\title{
Measurement of localized surface plasmon resonance of metallic nanostructures
}

\author{
Zhang Xingfang \\ College of Optoelectronics Engineering \\ Zaozhuang University \\ Zaozhuang, China \\ e-mail:zxf4114@126.com \\ Li Aiyun \\ College of Optoelectronics Engineering \\ Zaozhuang University \\ Zaozhuang, China \\ e-mail:aiyunli1980@126.com
}

\author{
Liu Fengshou \\ College of Optoelectronics Engineering \\ Zaozhuang University \\ Zaozhuang, China \\ e-mail: fsliu@126.com \\ Yan Xin \\ College of Optoelectronics Engineering \\ Zaozhuang University \\ Zaozhuang, China \\ e-mail: yanxinllj@163.com
}

\begin{abstract}
The measuring technique for characteristics of localized surface plasmon resonance (LSPR) effect of metallic nanostructures is studied in this paper. A simple apparatus for measuring the LSPR properties is designed and developed with the help of an integrating sphere using a single light beam; the sphere inner wall is coated with Teflon and has three ports used for sample, detector and incident light input. By changing the angle and position of the integrating sphere and the sample in the light path, the forward, backward and scattering spectroscopy of the metallic nanostructures can be obtained indicating that the absorption and scattering properties associated with the LSPR effect can be distinguished. It is founded that no matter what measurement form, the spectrum can reflect the general LSPR property, despite there is difference in the spectral intensity and peak. The measuring technique present in this paper may be an alternative way for the characterization of the LSPR effect.
\end{abstract}

Keywords-nanostructures; localized surface plasmon resonance; spectroscopy measurement; integrating sphere; reflection and transmission

\section{INTRODUCTION}

Noble metallic nanostructures have attracted extensive interest due to their remarkable optical phenomena caused by localized surface plasmon (LSP) resonances. When the incident electromagnetic wave is in resonance with the surface plasmon frequency of the nanoparticle, both a strong enhancement of local fields near the nanoparticle surface and a complex far-field spectrum consisting of both absorption and scattering contributions are produced. LSP resonance (LSPR) frequency is highly dependent on characteristics of the nanostructures such as size, shape, interparticle interval and dielectric environment around them [1,2]. This unique effect makes metallic nanoparticles promise in various potential applications, such as environmental, biochemical, medical science and other fields [3-6]. The LSPR peak position and intensity can be obtained by measuring the scattering or absorption spectroscopy of nanostructures. In general, for a single nanoparticle or nanostructures with very small size, the scattering properties are obtained using a dark field method [7], and for the macro-scale nanostructures, the reflective or transmissive spectroscopy are measured to characterize the LSPR properties by spectrometers and other conventional equipments [8,9]. However, the LSPR property consists of absorption contribution and scattering contribution. Complete understanding of scattering and absorption fields of metallic nanoparticles is of fundamental importance. Despite the great deal of theoretical work that has been done, few relevant experimental results have been reported. Due to the specular reflection and transmission light mixed together, it is difficult to distinguish between them and the scattering intensity and peak position can not be obtained directly from reflection or transmission spectra measured by the spectrometer. In recent years, more attention has paid to the direct measurement of the absorption and scattering properties $[10,11]$. D. D. Evanoff reported that extinction, scattering, and absorption cross sections and efficiencies were experimentally measured for chemically clean silver nanoparticles in water for 16 different sizes ranging from 29 to $136 \mathrm{~nm}$. An integrating spectrometer was designed to separate absorption and scattering contributions from the extinction spectra of nanoparticles. C. Langhammer obtained the optical characterization of four nanodisks using a UV-vis-near infrared spectrophotometer which records forward transmission and with a diffuse reflectance accessory, containing an integrating sphere detector, to measure the total scattering, absorption and scattering. In this paper, a LSPR measurement apparatus is designed with the help of an integrating sphere, the device structure is simple, and the scattering and absorption properties can be obtained by changing the angle and position of integrating sphere in the light path.

\section{APPARATUS}

The LSPR characteristics of nanostructures include two aspects: absorption and scattering. The absorption property can be obtained by measuring radiation light of 
the whole space before and after the incident light interaction with the nanostructures, and then deducting the substrate material absorption. As for the scattering property of nanostructures, which is spatially distributed, the forward and backward parts are mixed together with the specular reflection and transmission light, respectively. Therefore, the integrating sphere technique is applied to discriminate the two parts.

Fig .1 shows the home-built LSPR measurement device with an integrating sphere. The device consists of tungsten halogen light source, monochromator, detector, integrating sphere, etc. Tungsten halogen lamp (Newport 6334) radiation range is from $400 \mathrm{~nm}$ to $2400 \mathrm{~nm}$, the maximum output power is $250 \mathrm{~W}$, and the output instability is less than $0.1 \%$ in two hours. The broadband light output from lamp enters the monochromator (Zolix Omni- $\lambda$ 3007) with combined alignment lenses, the monochromator has three different gratings, and the scanning scope covers 200$2500 \mathrm{~nm}$. In order to eliminate the influence of high order diffraction, a filter wheel with 5 different cut-off wavelength filters is provided in the front of monochromator slit for automatic switching in the spectrum scanning process. The monochromatic light from the monochromator is then collimated by a lens group for the nanostructures measurement, the collimated spot size is about $5 \mathrm{~mm}$. Integrating sphere diameter is $50 \mathrm{~mm}$, the inner wall is coated with Teflon, the spectral reflectivity is up to $98 \%$ in the $400 \mathrm{~nm}-1800 \mathrm{~nm}$ range. Two ports A and $\mathrm{B}$ about $10 \mathrm{~mm}$ diameter are arranged on the both ends of the sphere surface along the diameter, as shown in Fig .1, and a $10 \mathrm{~mm}$ diameter port $\mathrm{C}$ is provided in the vertical direction. The three port openings area in integrating sphere is no more than 0.05 of the sphere surface area, it is the first rule to design an appropriate integrating sphere, because for port fractions larger than 0.05 , one begins to lose the advantage offered by the high reflectance coatings available for integrating spheres. The PTFE ethylene baffle is arranged inside the integrating sphere to avoid the reflection light directly into the detector. The Baffle can be considered extension of the sphere surface. Its contribution to the sphere area can be factored into the radiance equation although it is not usually significant. The fractional contribution of baffles to the sphere surface area is usually quite small. The light intensity of an arbitrary point in the sphere can be expressed as [12]

$$
I=\frac{I_{0}}{4 \pi R^{2}} \frac{\rho_{0}}{1-\rho_{w}\left(1-\sum_{i=0}^{n} f_{i}\right)-\sum_{i=0}^{n} \rho_{i} f_{i}}
$$

Where $\rho_{0}$ is the initial reflectance for incident flux, $\rho_{w}$ is the reflectance of the sphere wall, $\rho_{i}$ and $f_{i}$ are the reflectance and fractional port area of port opening $i$, respectively.

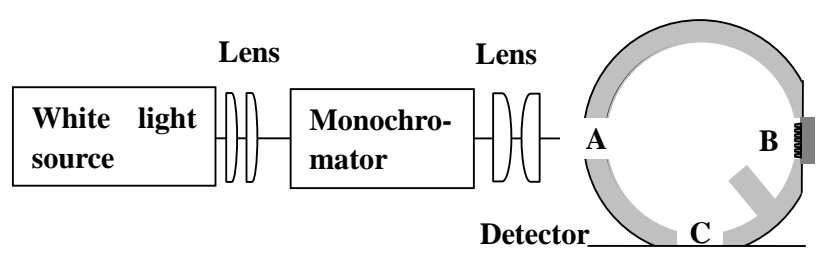

Figure1. Schematic configuration of the LSPR experimental system
The effective detection area of the detector $(\mathrm{Si})$ is 100 $\mathrm{mm}^{2}$, the response range is $200-1100 \mathrm{~nm}$. The absorption and scattering properties can be measured with the measurement device by rational using the integrating sphere. The sample used in the experiment is gold nanoshell arrays, which is fabricated by depositing gold onto a cleaned glass support covered with a close-packed monolayer of $200 \mathrm{~nm}$ diameter polystyrene (PS) spheres and subsequently by the removal of the PS spheres. The typical standard deviation in particle diameter is about $5 \%$ or smaller, and the multiple scattering contributions that make the total extinction be small.

\section{RESULTS AND DISCUSSION}

First, the absorption property of samples is tested. In experiment, the collimated light from port $\mathrm{A}$ inputs to the integrating sphere, the sample is placed inside the port B, where a tilt of the sample by $5^{\circ}$ from the normal assures that the specular scattering contribution and backward scattering is collected by the detector in the port $\mathrm{C}$. The measured signal of the sample is base line corrected with a reference plate, yielding more than $99 \%$ reflectance in the spectral range between 360 and $1200 \mathrm{~nm}$. Furthermore, a measured and base-line-corrected reflectance spectrum of a silica substrate without nanostructures is subtracted from the measured sample spectrum to exclude substrate contributions, the actual measured half space spectrum (referred to as backward spectroscopy) is shown in Fig .2. It has to be noted that some of the scattered light escapes through the input port $\mathrm{A}$ and thus leads to a slight underestimation of the scattering intensity, it is difficult to quantify due to the nonhomogenous spatial distribution of the scattered light from a decaying LSPR. According to the specification, the aperture areas of the incident ports correspond to $1 \%$ of the total area of the integrating sphere. As can be seen, a reflection valley and a reflection peak appear in about 500nm and $860 \mathrm{~nm}$, respectively. Then, forward scattering in the other half-space is collected with the sample placed on the front port of the integrating sphere, the collimated light from port B inputs to the integrating sphere, the sample is still placed in port B facing the direction of incident light, the transmitted and forward scattering light can be received by the detector in port $\mathrm{C}$ after adjusting the position and angle of the integrating sphere. The measured spectrum including transmission and forward scattering contributions (referred to as forward spectrum) is also base line corrected with a reference plate, which is shown in Fig .2. The spectrum also deducts the substrate contributions. After comparison with the forward and backward spectra in Fig .2, we can see that the reflection peak and valley in the two spectra have the same positions, but have the opposite directions. The peak position in $860 \mathrm{~nm}$ is the LSPR peak, which is caused by free electron collective oscillation in the gold nanostructures, and the peak in $500 \mathrm{~nm}$ origins from the spectral superposition of $\mathrm{sp}-\mathrm{d}$ interband resonance transitions of $5 \mathrm{~d}-6 \mathrm{~s}$ and intraband resonance transitions of 6 s for gold material $[9,13]$. 


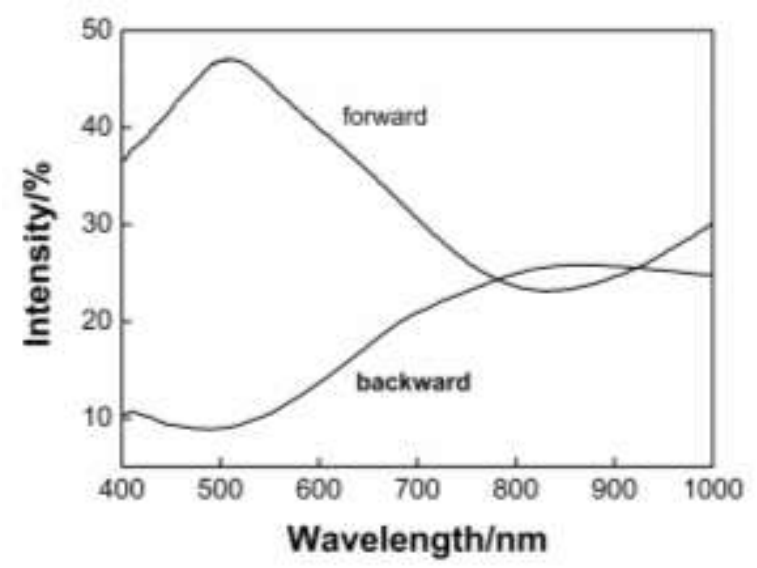

Figure 2. Forward and backward spectrum of nanostructures

The sum of forward and backward spectra thus represents the total amount of light scattered by a sample in all spatial directions, and the absorption intensity can be calculated, as shown in Fig .3. The absorption peak of the sample is about $810 \mathrm{~nm}$, and the minimum absorption is in $500 \mathrm{~nm}$. Compared with the spectral line in Fig .2, absorption and backward spectral peak positions just located at the two sides of the forward spectrum peak position, the peak distribution is similar to the theoretical results [14]. It is thus important to note that the peak distribution, in principal, represent single particle properties. However, polydispersity in the particle size distribution leads to inhomogeneous broadening of the spectra. The experimentally determined absolute values for inhomogeneous broadening contributions are published elsewhere [15]. Thus the measured optical property for a given quoted size is an average property over the particle size distribution on the sample. However, we do not expect this effect to significantly influence the spectrum obtained here, since the typical standard deviation in particle diameter is about $5 \%$ or smaller.

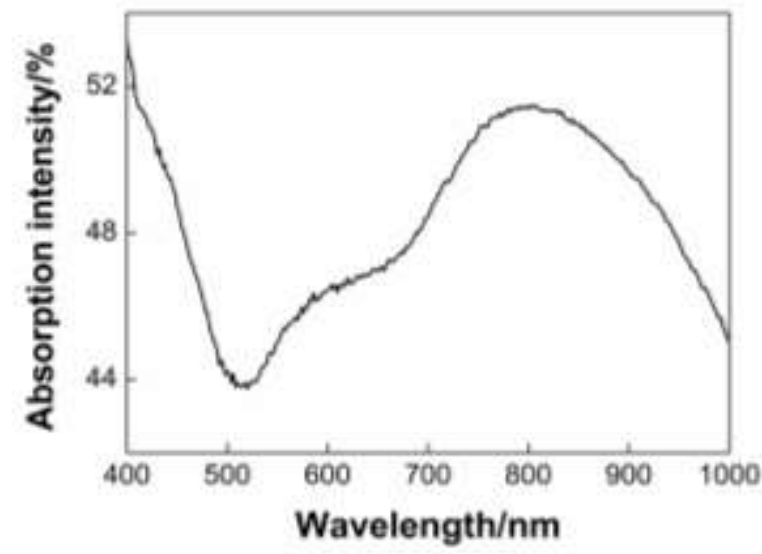

Figure 3.Measured absorption spectroscopy

The scattered light contains two parts: backward scattering and forward scattering. The backscatter property is first measured, the collimated light from port A inputs to the integrating sphere, the sample is placed the port B inside integrating sphere, and the specular reflection escapes from the port A of integrating sphere. Thus only the backscattering component of the backward scattered light is received by the dtector in port $C$. The backscattering spectroscopy is shown in Fig .4. The backscattering spectral peak is about $830 \mathrm{~nm}$, the scattering intensity is slightly higher than $7 \%$. Compared with the backward spectrum in Fig .2, two spectra have similar line shape, but the reflection peak position is different about $30 \mathrm{~nm}$, this is probably caused by different incident angle in the two measurements [16]. The forward scattering spectroscopy can be obtained as follows. The collimated light from port $\mathrm{B}$ inputs to the integrating sphere, the sample is still placed in the port $\mathrm{B}$, the transmission light escapes from port A by adjusting the sample orientation. Thereby the forward scattering can be measured in port $\mathrm{C}$, after deduction of the substrate absorption and background noise processing, the spectrum curve is shown in Fig .5. Can be seen from the graph, the spectral intensity increases as the incident wavelength increases, but the reflection peak wavelength is not obvious in the near infrared, mainly the forward scattering light is very weak.

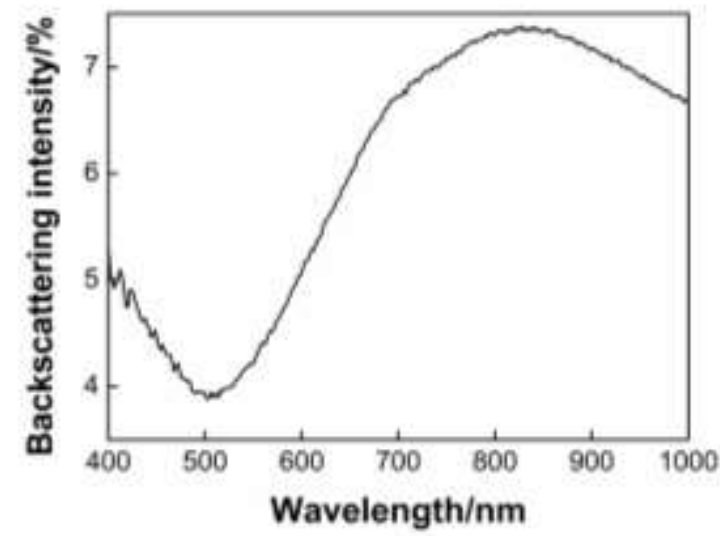

Figure 4. Measured backward scattering spectroscopy

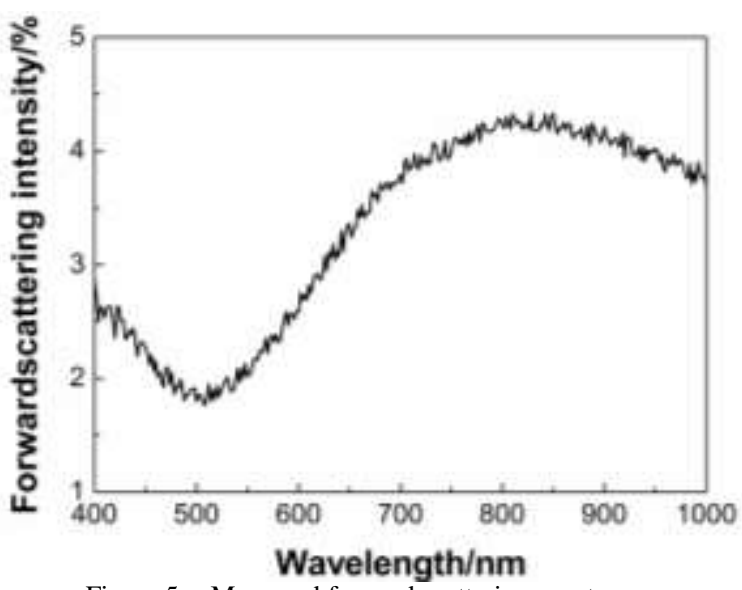

Figure 5. Measured forward scattering spectroscopy

Fig .6 shows the total scattering spectra. The scattering spectral peak is in $870 \mathrm{~nm}$, the intensity is about $13 \%$. Comparison with forward scattering and backward scattering spectra, spectral characteristics is mainly similar to the backward scattering spectrum, but its peak position is closer to the back reflection spectral peak position. If the total contribution from scattering and absorption is considered, the LSPR spectrum can be obtained, as shown in Fig .7. The LSPR peak position is about 820nm, the intensity is $60 \%$. When the incident light interacts with the sample, $13 \%$ of the energy converted into light scattering, while $47 \%$ of the energy is converted into heat loss. The LSPR intensity is found to be dominated almost entirely by 
absorption part. The reason is that the thickness of the nanoshell particles in the sample is very thin, only tens of nanometers, so the absorption is very large. With increasing nanoshell diameter the scattering contribution to the total LSPR will become more and more significant until it finally starts to dominate for particles with a thickness larger than $50 \mathrm{~nm}$. Similar trends have been observed for spherical metaliic nanoparticles in colloidal solution. Comparison with the transmission and reflection spectra, no matter what measurement form, the spectrum can reflect the general LSPR property, but there is difference in the spectral intensity and peak.

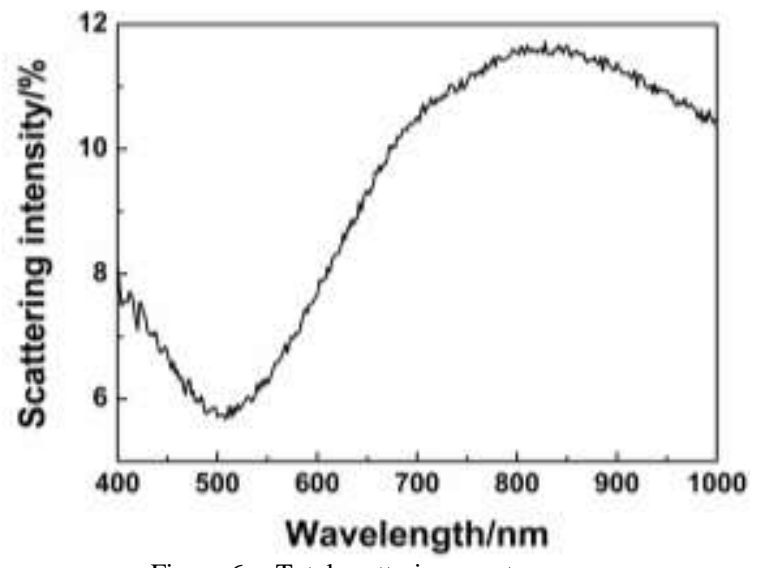

Figure 6. Total scattering spectroscopy

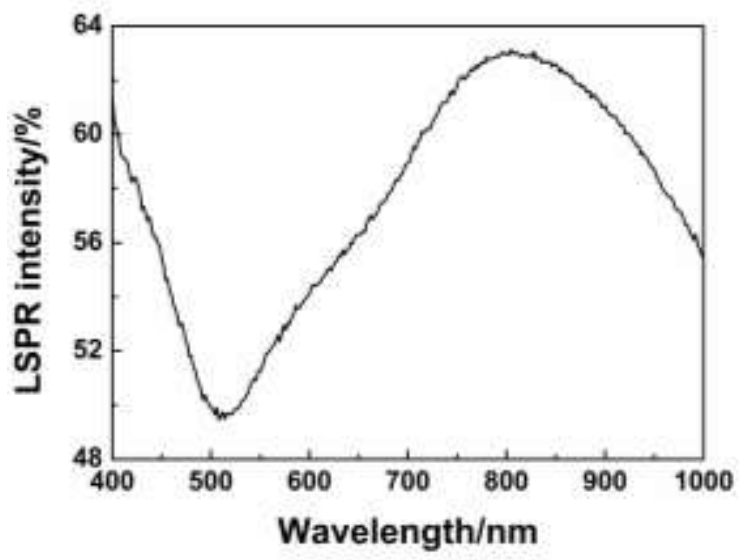

Figure 7. LSPR spectroscopy

\section{CONLUSIONS}

A simple device including tungsten halogen light source, monochromator, detector and integrating sphere with three ports, is developed for measuring LSPR characteristics using a single light beam. By changing the position and angle of the integrating sphere in the light path, the absorption, forward and backward scattering spectra of nanostructures can be well measured. The results show that the scattering and absorption spectra can be districted by using this home-built device with rational using the integrating sphere. This may provide a new way for measuring LSPR characteristics.

\section{ACKNOWLEDGMENT}

This work was financially supported by the National Natural Science Foundation (11247237), Zaozhuang Science and Technology Plan (201343), Doctoral Fund of Zaozhuang University, scientific research and innovation team "Zaozhuang University optoelectrical science and technology" project, and and College Scientific Research Fund of Zaozhuang University (2014YY04).

\section{REFERENCES}

[1] D. R. Willett and G. Chumanov, "LSPR Sensor Combining Sharp Resonance and Differential Optical Measurements," Plasmonics, Vol. 9, Aug. 2014, pp. 1391-1396, doi: 10.1007/s11468-0149754-8.

[2] J. R. Krenn, H. Ditlbacher, G. Schider, et al., "Surface plasmon micro- and nano-optics," Journal of Microscopy, vol. 209, Mar. 2003, pp. 167-172, doi: 10.1046/j.1365-2818.2003.01088.x.

[3] X. Zhang and Z. Liu, "Superlenses to overcome the diffraction limit," Nature Materials, vol. 7, Jun. 2008, pp. 435-441, doi:10.1038/nmat2141.

[4] W. Cai and A. P. Vasudev and M. L. Brongersma, "Electrically controlled nonlinear generation of light with plasmonics," Science, vol. 333, Sep. 2011, pp. 1720-1723, doi: 10.1126/science.1207858.

[5] W. A. Murray and W. L. Barnes, "Plasmonic materials," Advanced Materials, vol. 19, Oct. 2007, pp. 3771-3782, doi: 10.1002/adma.200700678.

[6] H. Im, K. C. Bantz, S. H. Lee, et al., "Self - Assembled Plasmonic Nanoring Cavity Arrays for SERS and LSPR Biosensing," Advanced Materials, vol. 25, Feb. 2013, pp. 2678-2685, doi: 10.1002/adma.201204283.

[7] C. M. Hill, R. Bennett, C. Zhou, et al., "Single Ag Nanoparticle Spectroelectrochemistry via Dark Field Scattering and Fluorescence Microscopies," The Journal of Physical Chemistry C, vol. 119, Mar. 2015, pp. 6760-6768, doi: 10.1021/jp511637a.

[8] J. S. G. Bouillard, W. Dickson, D. P. Oconnor, et al., "Lowtemperature plasmonics of metallic nanostructures," Nano Letters, vol. 12, Feb. 2012, pp.1561-1565, doi: 10.1021/nl204420s.

[9] T. Ung, L. M. Liz-Marzan, P. Mulvaney, "Gold nanoparticle thin films," Colloids and Surfaces A, vol. 202, Apr. 2002, pp. 119-126, doi: S0927-7757(01)01083-4.

[10] D. D. Evanoff and G. Chumanov, "Size-controlled synthesis of nanoparticles.2. measurement of extinction, scattering, and absorption cross sections," Journal of Physical Chemistry B, vol. 108, Aug. 2004, pp. 13957-13962, doi: 10.1021/jp0475640.

[11] C. Langhammer, B. Kasemo, I. Zoric, "Absorption and scattering of light by Pt, Pd, Ag, and Au nanodisks: Absolute cross sections and branching ratios," Journal of Chemical Physics, vol. 126, May 2007, pp. 194702, doi: 10.1063/1.2734550.

[12] http://www.bfioptilas.com/objects/52_23_2079453411/Guide-toIntegratingSphereRadiometry\&Photometry.pdf

[13] J. T. Seo, Q. Yang, W. J. Kim, "Optical nonlinearities of Au nanoparticles and Au/Ag coreshells," Optics Letters, vol. 34, Feb. 2009, pp. 307-309, doi: 10.1364/OL.34.000307.

[14] J. B. Lassiter, M. W. Knight, N. A. Mirin, et al., "Reshaping the plasmonic properties of an individual nanoparticle," Nano Letters, vol, 9, Sep. 2009, pp. 4326-4332, doi: 10.1021/n19025665.

[15] C. Langhammer, Z. Yuan, I. Zoric, et al., "Plasmonic properties of supported Pt and Pd nanostructures," Nano Letters, vol.6, Mar. 2007, pp. 833-838, doi: 10.1021/n1060219x

[16] C. Charnay, A. Lee, S. Q. Man, et al., "Reduced symmetry metallodielectric nanoparticles: chemical synthesis and plasmonic properties," Journal of Physical Chemistry B, vol. 107, Jun. 2003, pp. 7327-7333, doi: 10.1021/jp034309r. 